\title{
On the effects of vertical air velocity on winter precipitation types
}

\author{
J. M. Thériault and R. E. Stewart \\ Department of Atmospheric and Oceanic Sciences, 805 Sherbrooke St. West, McGill University, Montreal, H3A 2K6, Canada
}

Received: 30 August 2006 - Revised: 14 February 2007 - Accepted: 22 February 2007 - Published: 21 March 2007

\begin{abstract}
The various precipitation types formed within winter storms (such as snow, wet snow and freezing rain) often lead to very hazardous weather conditions. These types of precipitation often occur during the passage of a warm front as a warm air mass ascends over a cold air mass. To address this issue further, we used a one-dimensional kinematic cloud model to simulate this gentle ascent $(\leq 10 \mathrm{~cm} / \mathrm{s})$ of warm air. The initial temperature profile has an above $0^{\circ} \mathrm{C}$ inversion, a lower subfreezing layer, and precipitation falls from above the temperature inversion. The cloud model is coupled to a double-moment microphysics scheme that simulates the production of various types of winter precipitation. The results are compared with those from a previous study carried out in still air. Based on the temporal evolution of surface precipitation, snow reaches the surface significantly faster than in still air whereas other precipitation types including freezing rain and ice pellets have a shorter duration. Overall, even weak background vertical ascent has an important impact on the precipitation reaching the surface, the time of the elimination of the melting layer, and also the evolution of the lower subfreezing layer.
\end{abstract}

\section{Introduction}

Various precipitation types occur in the transition region of winter storms which is bounded by rain on one side and snow on the other (Stewart, 1992). Between the limits defined by the snow and rain, many types of winter precipitation are commonly observed such as ice pellets, freezing rain and freezing drizzle as well as particles composed of both liquid and solid. In particular, precipitation types containing liquid (such as freezing rain and wet snow) can lead to catastrophic icing events when falling on subfreezing surfaces. A good example is the 1998 Ice Storm in Montreal, the

Correspondence to: Julie M. Thériault

(julie.theriault@mcgill.ca) most catastrophic weather event in Canadian history (Henson et al., 2007).

The transition region of a winter storm is often associated with a warm front. The warm air mass replacing the cold air mass is a favorable weather situation for the formation of a temperature inversion. This type of temperature profile, consisting of a warm layer of temperature $>0{ }^{\circ} \mathrm{C}$ aloft and a subfreezing layer below it, is required to form some of the hazardous types of precipitation (e.g. Wagner, 1957; Zerr, 1997). For example, snowflakes falling through it will melt or partially melt, depending on their size and atmospheric conditions in the warm layer. Depending on the degree of melting, these particles may or may not refreeze into different types of precipitation such as freezing rain and ice pellets when falling into the subfreezing layer.

Phase changes associated with such melting and freezing will influence the temperature profile and the ensuing precipitation types. For instance, the melting of frozen particles has a very important impact on the atmosphere (Findeisen, 1940). In a saturated environment, when the temperature is greater than $0^{\circ} \mathrm{C}$, heat is extracted from the atmosphere because of melting. This process occurs until the temperature reaches $0^{\circ} \mathrm{C}$. At that temperature, melting stops, thus the melting layer evolves into an isothermal layer of $0^{\circ} \mathrm{C}$.

From a dynamical point of view, common factors such as horizontal temperature advection and vertical air velocity can alter the temperature structure, hence, changing the precipitation types. For example, vertical air velocity changes the temperature by adiabatic warming and cooling of the air through descent and ascent, respectively. Such changes in temperature also generate subsaturated and supersaturated conditions in the atmosphere which will also alter the precipitation types through variation of warming and cooling rates. Such ascent is always associated with the warm air mass of the warm conveyor belt gently rising over the cold air mass of a cyclonic storm (Stewart and Macpherson, 1989). A schematic of this feature is shown Fig. 1.

Published by Copernicus GmbH on behalf of the European Geosciences Union. 


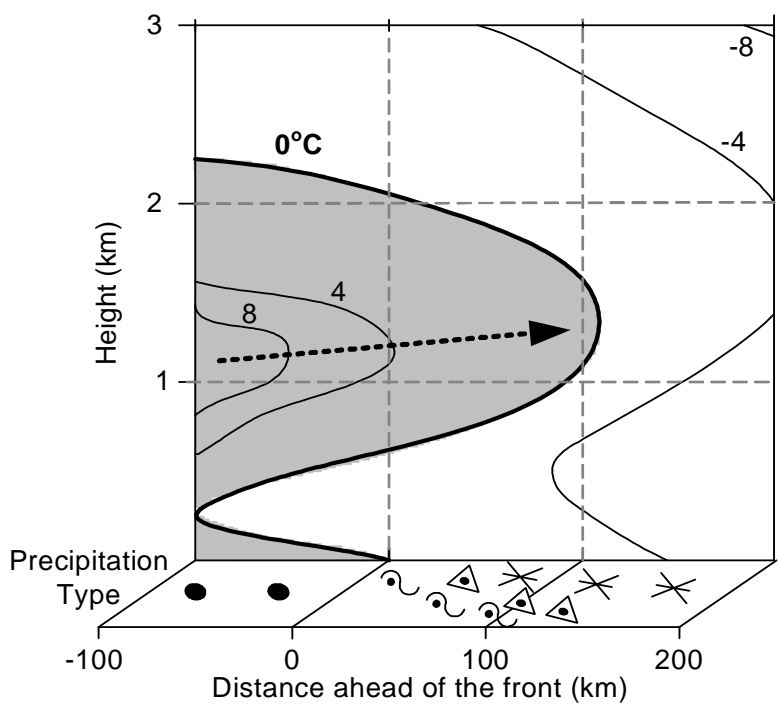

Snow • Rain $\frown$ Freezing Rain $\triangle$ Ice Pellets

Fig. 1. A cross-section of a typical warm front of a winter storm that includes a precipitation transition region (adapted from Stewart et al., 1995). The gray shading and the dotted arrow represent the warm air mass gently flowing above a cold air mass during the passage of a warm front. The cold air is ahead of the surface front and the warm air is behind it. The isotherms are the solid lines. A distribution of surface precipitation types is also indicated on the diagram.

Thériault et al. (2006) conducted a study on the formation of various types of winter precipitation in still air. However, we recognize that the weak upward air velocity in the warm conveyor belt influences the types and duration of precipitation in the atmosphere and at the surface. However, no systematic study has ever been conducted on this issue.

Given the importance of vertical air velocity, we will begin to address this issue by carrying out a systematic study of the influence of background ascending air associated with a warm front on the formation of winter precipitation types.

The paper consists of a description of the one-dimensional kinematic cloud model and the microphysics scheme used in this study (Sect. 2) and an explanation of the experimental design (Sect. 3). We compare the precipitation types formed in still air and in a background ascending air environment using a typical temperature profile (Sect. 4). In the same section, we show the impact of different vertical air velocities on the formation of precipitation. Finally, concluding remarks are given in Sect. 5.

\section{Model description}

A systematic study on the effect of vertical air velocity on precipitation types and their duration has been performed using a one-dimensional kinematic cloud model coupled to the double-moment version of the multi-moment bulk microphysics scheme described in Milbrandt and Yau (2005a,b) and improved for winter precipitation types by Thériault et al. (2006).

The one-dimensional cloud model has been developed by Milbrandt and Yau (2005a,b) to test their multi-moment microphysics scheme. The model is briefly described in Milbrandt and Yau (2005b). However, we will describe it in greater detail in the following section.

\subsection{One-dimensional cloud model}

The one-dimensional kinematic cloud model is initialized with vertical $(\mathrm{z})$ profiles of temperature $(\mathrm{T})$, dew point temperature $\left(\mathrm{T}_{d}\right)$ when $\mathrm{T}>0^{\circ} \mathrm{C}$ and frost point temperature $\left(\mathrm{T}_{f}\right)$ when $\mathrm{T}<0^{\circ} \mathrm{C}$. There are 100 vertical levels evenly spaced over a column of $3 \mathrm{~km}$ and we assume hydrostatic balance.

The cloud model solved the balance equation for the mass mixing ratio and total concentration. The general form of the equation is

$\frac{\partial Y_{x}}{\partial t}+\vec{\nabla} \cdot\left(Y_{x} \vec{U}\right)=\left.\frac{\partial Y_{x}}{\partial t}\right|_{S}$

where $Y_{x}$ is the mass mixing ratio $\left(\rho q_{x}\right)$, total concentration $\left(N_{x}\right)$ for each hydrometeor category $\mathrm{x}$ that is predicted by the scheme (Sect. 2.2), $\vec{U}$ is the three-dimensional air velocity, $\mathrm{S}$ is the sources and sinks term calculated by the doublemoment microphysics scheme (Milbrandt and Yau, 2005b; Thériault et al., 2006). The sources and sinks term also includes precipitation sedimentation.

Considering only air velocity in the vertical and using the anelastic approximation (i.e. density is varying only with height), the balance equation (Eq. 1) for the mass mixing ratio and total concentration is reduced to

$\frac{\partial q_{x}}{\partial t}=-q_{x}\left(\frac{\partial w}{\partial z}+w \frac{\partial \ln \rho}{\partial z}\right)-w \frac{\partial q_{x}}{\partial z}+\left.\frac{1}{\rho} \frac{\partial q_{x}}{\partial t}\right|_{S}$,

and

$\frac{\partial N_{x}}{\partial t}=-N_{x} \frac{\partial w}{\partial z}-w \frac{\partial N_{x}}{\partial z}+\left.\frac{\partial N_{x}}{\partial t}\right|_{S}$

where $w$ is the vertical air velocity and is a function of height and time (Eq. 11), $\rho$ is the air density.

In the mass mixing ratio equation (Eq. 2) the first term on the right hand side of the equation is divergence, the second term is stratification, the third term is advection and the fourth term is the microphysical sources and sinks as well as sedimentation calculated by the double moment microphysics scheme. In the total concentration equation (Eq. 3), the first term is divergence, the second is advection and the third term represents the microphysical sources and sinks as well as sedimentation computed in the double-moment microphysics scheme. 
Table 1. Definition of various winter precipitation types.

\begin{tabular}{|c|c|}
\hline Precipitation types & Definition \\
\hline Rain $^{\mathrm{a}}$ & $\begin{array}{l}\text { Precipitation in the form of liquid water drops that have diameters greater than } 0.5 \mathrm{~mm} \text {, or, } \\
\text { if widely scattered, the drops may be smaller. }\end{array}$ \\
\hline Freezing rain ${ }^{\mathrm{a}}$ & $\begin{array}{l}\text { Rain that falls in liquid form but freezes upon impact to form a coating of glaze upon the } \\
\text { ground and on exposed objects. }\end{array}$ \\
\hline Snow $^{\mathrm{a}}$ & $\begin{array}{l}\text { Precipitation composed of white or translucent ice crystals, chiefly in complex branch } \\
\text { hexagonal form and often agglomerated into snowflakes. }\end{array}$ \\
\hline Ice pellets ${ }^{\mathrm{a}}$ & $\begin{array}{l}\text { A type of precipitation consisting of transparent or translucent pellets of ice, } 5 \mathrm{~mm} \text { or less } \\
\text { in diameter. }\end{array}$ \\
\hline Graupel $^{\mathrm{a}}$ & $\begin{array}{l}\text { Heavily rimed snow particles, often called snow pellets. Sometimes distinguished by shape } \\
\text { into conical, hexagonal, and lump (irregular) graupel. }\end{array}$ \\
\hline Wet snow ${ }^{\mathrm{a}}$ & $\begin{array}{l}\text { Snow that contains a great deal of liquid water. If free water entirely fills the air space in } \\
\text { the snow it is classified as "very wet" snow. }\end{array}$ \\
\hline Refrozen wet snow ${ }^{b}$ & Refrozen wet snowflake. \\
\hline Slush $^{\mathrm{b}}$ & $\begin{array}{l}\text { Precipitation composed of a mixture of liquid and ice in which the original snowflake's } \\
\text { shape is not discernable. }\end{array}$ \\
\hline Ice crystals ${ }^{\mathrm{c}}$ & Pristine ice crystals. \\
\hline Cloud droplets ${ }^{\mathrm{c}}$ & Small non-sedimenting water droplets. \\
\hline
\end{tabular}

a from Glickman (2000)

$\mathrm{b}$ from Thériault et al. (2006)

c from Milbrandt and Yau (2005b)

The temperature is a crucial parameter in the model. The general form of the temperature equation is

$$
\frac{\partial T}{\partial t}+\vec{\nabla} \cdot(T \vec{U})=\left.\frac{\partial T}{\partial t}\right|_{S},
$$

where $\mathrm{T}$ is the temperature, $\vec{U}$ is the velocity field and $\mathrm{S}$ is the source and sinks term from the microphysics scheme and from adiabatic ascent.

Our model is restricted to a vertical air column, thus the temperature equation is reduced to

$$
\frac{\partial T}{\partial t}=-T \frac{\partial w}{\partial z}+w\left(\Gamma_{m}-\frac{\partial T}{\partial z}\right)+\left.\frac{L}{c_{p}} \frac{\partial q_{x}}{\partial t}\right|_{S},
$$

where $\Gamma_{m}$ is the pseudoadiabatic lapse rate $\left(\Gamma_{m}=-6.5^{\circ} \mathrm{C} / \mathrm{km}\right), \mathrm{L}$ is the latent heat of fusion, evaporation or sublimation, $c_{p}$ is the heat capacity, $\mathrm{S}$ represents the microphysical sources and sinks calculated in the double-moment microphysics scheme.

We have set the divergence term in Eqs. (2), (3) and (5) to zero because, in this particular experiment, the rising air is not related to divergence but to a warm air mass replacing a cold air mass. Thus, we consider only the effect of vertical advection, stratification (only in Eq. 2) and the microphysical sources and sinks including precipitation sedimentation. Thus, the mass mixing ratio balance equation is

$$
\frac{\partial q_{x}}{\partial t} \simeq-q_{x} w \frac{\partial \ln \rho}{\partial z}-w \frac{\partial q_{x}}{\partial z}+\left.\frac{1}{\rho} \frac{\partial q_{x}}{\partial t}\right|_{S},
$$

the total concentration balance equation is

$$
\frac{\partial N_{x}}{\partial t} \simeq-w \frac{\partial N_{x}}{\partial z}+\left.\frac{\partial N_{x}}{\partial t}\right|_{S}
$$

and the temperature equation is

$$
\frac{\partial T}{\partial t} \simeq w\left(\Gamma_{m}-\frac{\partial T}{\partial z}\right)+\left.\frac{L}{c_{p}} \frac{\partial q_{x}}{\partial t}\right|_{S},
$$

The terms are computed at every time step for every vertical level in the air column. Also, for the mass mixing ratio and total concentration they are computed for every category of hydrometeor in the microphysics scheme. After the calculation of the stratification term as well as the advection and adiabatic ascent, the microphysics scheme is called by the cloud model and all the microphysical processes and sedimentation are computed. The cloud model forces mass conservation within the column and the advection of precipitation is computed using the Euler method. The double-moment microphysics scheme is described in the following section.

\subsection{Double-moment microphysics scheme}

The double-moment microphysics scheme predicts two moments of the size distribution of all the hydrometeors defined in the scheme (Milbrandt and Yau, 2005a,b). The size distribution of each hydrometeor category $\mathrm{x}$ is represented by an analytic function:

$N_{x}\left(D_{x}\right)=\int_{0}^{\infty} N_{0 x} D_{x}^{\alpha_{x}} \exp \left(-\lambda_{x} D_{x}\right) d D_{x}$ 
In the Melting Layer

(a)

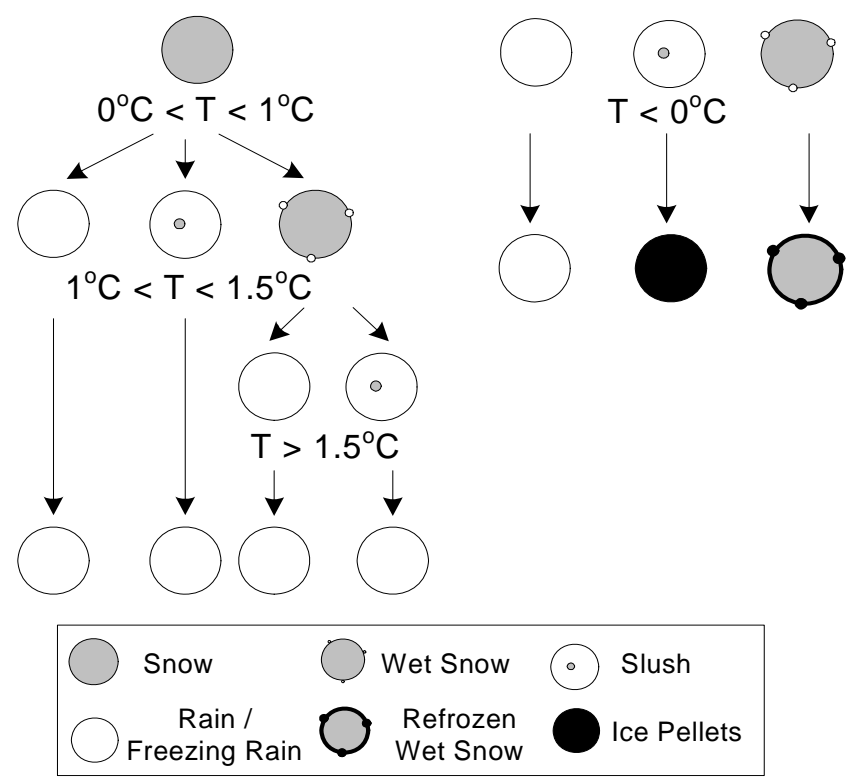

Fig. 2. A schematic of the different types of precipitation formed under various temperatures. (a) The melting of snowflakes into slush, wet snow and rain within the melting layer. Wet snow melts into rain and slush and eventually slush melts into rain. (b) The refreezing of liquid and partially liquid particles. The rain changes into freezing rain, slush into ice pellets and wet snow into refrozen wet snow within the subfreezing layer below the melting layer.

where $D_{x}$ is the diameter of hydrometeor category $\mathrm{x}$, $N_{x}\left(D_{x}\right)$ is the concentration of particles size $D_{x}, N_{0 x}$ is a constant, $\lambda_{x}$ is the slope of the distribution and $\alpha_{x}$ is the shape parameter.

The moment equation is

$M_{x}(n)=\int_{0}^{\infty} D_{x}^{n} N_{x}\left(D_{x}\right) d D_{x}$

where the $M_{x}(n)$ is the nth moment of the size distribution (Eq. 9). Thus, the two moments predicted by the scheme are the zeroth moment $(n=0)$ which gives the total number concentration and the third moment $(\mathrm{n}=3)$ which is proportional to the mass mixing ratio for each category $\mathrm{x}$.

The size distribution parameters, $N_{0 x}$ and $\lambda_{x}$, are diagnosed as a function of the mixing ratio and the total concentration at every level for each time step and hydrometeor category $\mathrm{x}$. The shape parameter, $\alpha_{x}$, is diagnosed as a function of the mean mass diameter (Milbrandt and Yau, 2005b).

The hydrometeor categories predicted in the scheme are cloud droplets, rain, freezing rain, snow, ice crystals, graupel, ice pellets, slush, wet snow and refrozen wet snow and they are defined in Table 1. Milbrandt and Yau (2005b) describes the microphysical sources and sinks for particles including cloud droplets, rain, snow, ice crystals, graupel and hail. Some of the processes associated with hail production also apply to ice pellets. They also described the temperature tendency due to the microphysical sources and sinks associated with those hydrometeors. Sedimentation is also computed for each precipitation category. Their bulk densities, mass-diameter relation and terminal velocity-diameter relation are given in Milbrandt and Yau (2005a,b).

The semi-melted particle categories including slush and wet snow are described in Thériault et al. (2006). Figure 2 shows a schematic of the evolution of those precipitation types within the melting layer and within the subsequent subfreezing layer. We assume the same bulk densities, massdiameter relation and velocity-diameter relation as the rain category for slush and as the snow category for wet snow. Thus the main difference between rain and slush is whether or not the particle will refreeze when falling at temperatures near $0^{\circ} \mathrm{C}$. When falling into the subfreezing layer below the melting layer, slush will refreeze into ice pellets because we assume a small amount of ice remaining in the particle. This tiny amount of ice within the particle will trigger the freezing whereas a completely liquid drop rarely refreezes at the relatively warm temperatures typically found in the transition region $\left(\mathrm{T}>-6^{\circ} \mathrm{C}\right)$. For wet snowflake, it will refreeze into refrozen wet snow because we assume a small amount of liquid within it. Finally, rain is changed into freezing rain in the subfreezing layer.

When various precipitation types undergo phase changes, latent heat is released in the atmosphere, changing the environmental temperature and moisture. For example, when snow melts into rain, latent heat is extracted from the atmosphere and it is called cooling-by-melting. This cooling in saturated conditions will lead to supersaturation and this leads to cloud droplet formation. The condensation will release heat and increase the temperature. Finally, when semiliquid or liquid drops freeze, latent heat is released, warming the environment and this is called warming-by-freezing.

Collisions between particles of different phases can also alter the temperature profile and may also lead to changes in the types. Collisions between, for example, liquid drops and particles containing ice in the subfreezing region will lead to the freezing of the liquid particles. All the collision processes are explained in Milbrandt and Yau (2005b).

Finally, the sedimentation is explained in detail in Milbrandt and Yau (2005a). They showed that sedimentation in a double-moment microphysics scheme using the diagnosed shape parameter, $\alpha$, is comparable to an analytic bin model.

\subsection{Prescribed vertical air velocity}

Weak background ascending air is prescribed in the melting layer only and this is consistent with the observed structure of warm frontal regions (Fig. 1). We have assumed a halfsine wave as in Milbrandt and Yau (2005b) with a maximum vertical air velocity in the middle of the melting layer. At the top and bottom of the melting layer, the vertical velocity is 
zero, as well as in the subfreezing layers above and below the melting layer. Thus the vertical air velocity equation is

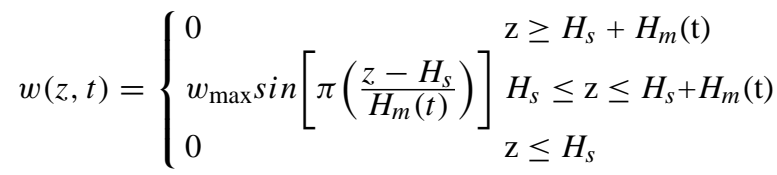

where $w_{\max }$ is the maximum vertical velocity prescribed in the middle of the melting layer, $H_{S}$ is the depth of the subfreezing layer, $\mathrm{z}$ is the height (Fig. 3), $H_{m}(t)$ is the depth of the melting layer varying with time due to the phase changes occurring within it (Sect. 2.2).

The vertical velocity is prescribed by Eq. (3) and it is calculated at every time step until the temperature through the entire original melting layer region is less than $0.01^{\circ} \mathrm{C}$. At that stage, the vertical air velocity is set to zero. Thus, the top of the melting layer will decrease in height over time due to the absorption of latent heat by melting (cooling-by-melting) and adiabatic cooling. Thus, the depth of the melting layer will change with time $\left(H_{m}(t)\right)$.

\section{Experimental design}

A winter storm precipitation transition region is simulated using the one-dimensional kinematic cloud model coupled to a double-moment microphysics scheme.

The model is initialized with a temperature profile composed of a melting layer aloft associated with ascending air and a subfreezing layer below it with no vertical air velocity. During the time evolution, the melting layer evolves into an isothermal layer of $0^{\circ} \mathrm{C}$ due to the microphysical processes occurring in that region, as well as the adiabatic cooling from ascending air. For example, the time evolution of such a temperature profile could be associated with the temperature profile changing from $50 \mathrm{~km}$ ahead of the front to $100 \mathrm{~km}$ ahead of the front (Fig. 1). This situation is often associated with a horizontal variation of precipitation types at the surface as also shown in Fig. 1 (Stewart, 1992). However, the goal of this paper is to study the general effects of background vertical air velocity on precipitation types and not reproduce a specific event.

To initialize the cloud model, we used the same typical temperature and humidity profile, as well as the same precipitation rate, as in Thériault et al. (2006). This allowed us to compare the results in still air with those obtained including the effects of background vertical air velocity. We call it a typical temperature profile because similar ones have been observed during mixed-phase precipitation events (Hanesiak and Stewart, 1995; Zerr, 1997). In Fig. 3, the typical temperature profile extends up to $3 \mathrm{~km}$ above the surface with a surface temperature of $-6^{\circ} \mathrm{C}\left(T_{\text {surface }}\right)$. The maximum temperature in the inversion is $2^{\circ} \mathrm{C}\left(T_{\max }\right)$ at $2 \mathrm{~km}$. The depth of the melting layer $\left(H_{m}(0)\right)$ is $1 \mathrm{~km}$ and the depth of the subfreezing layer $\left(H_{S}\right)$ is $1.5 \mathrm{~km}$. The air associated with that

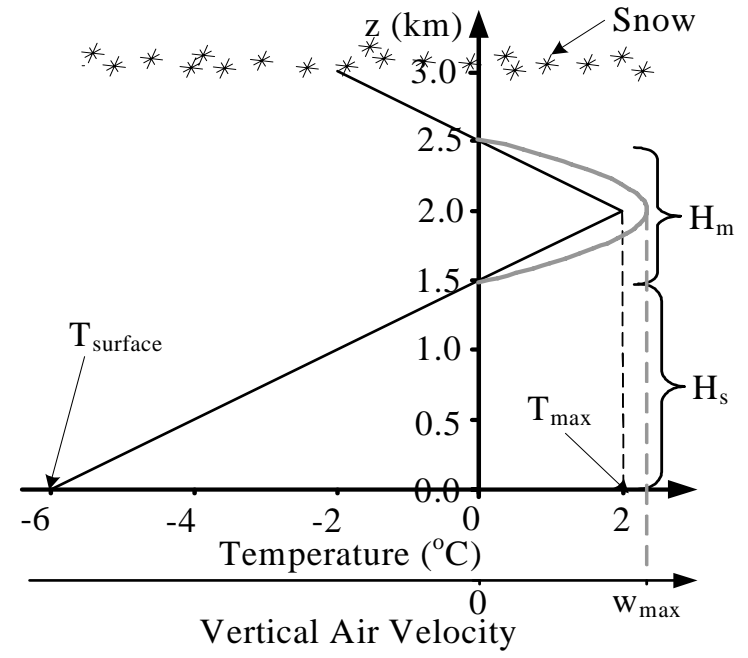

Fig. 3. A schematic diagram of the typical temperature and humidity profile used for this study is shown. $H_{m}$ is the depth of the melting layer $\left(\mathrm{T}>0^{\circ} \mathrm{C}\right), H_{S}$ is the depth of the subfreezing layer $\left(\mathrm{T}<0^{\circ} \mathrm{C}\right), T_{\max }$ and $T_{\text {surface }}$ are the maximum and surface temperatures of the temperature profile. The initial profile of weak ascending air (Eq. 11) from the warm conveyor belt is shown in grey with the maximum vertical air velocity in the middle of the melting layer $\left(w_{\max }\right)$. Snow is falling at $5 \mathrm{~mm} / \mathrm{h}$ from $3 \mathrm{~km}$ above the surface.

temperature profile is saturated with respect to water at temperature $>0^{\circ} \mathrm{C}$ and with respect to ice at temperature $<0^{\circ} \mathrm{C}$. The initial vertical motion profile (Eq. 11) is also shown on Fig. 3.

It should be noted that changing the temperature profile will lead to slightly different but very similar results (Thériault et al., 2006) but the goal of this paper is to demonstrate the sensitivity of the environmental temperature and the ensuing precipitation types to a weak background vertical air velocity on. Since the cloud model is one-dimensional, the dynamics needed to develop a warm front and the temperature inversion is not included into this cloud model. That is the reason why a temperature profile with a melting layer is used to initialize the model.

As snow falls into the melting layer, phase changes will occur, and it may evolve into various types of particles. We studied several values of $w_{\max }: 0 \mathrm{~cm} / \mathrm{s}, 2 \mathrm{~cm} / \mathrm{s}, 4 \mathrm{~cm} / \mathrm{s}$, $5 \mathrm{~cm} / \mathrm{s}, 6 \mathrm{~cm} / \mathrm{s}, 8 \mathrm{~cm} / \mathrm{s}$ and $10 \mathrm{~cm} / \mathrm{s}$. These are typical values inferred from observations during the passage of many warm fronts (Szeto and Stewart, 1997).

The following section illustrates the effect of background vertical air ascent on the evolving temperature profile with time, the mass content of precipitation types formed, the surface precipitation evolution with time and the accumulated precipitation at the surface. 

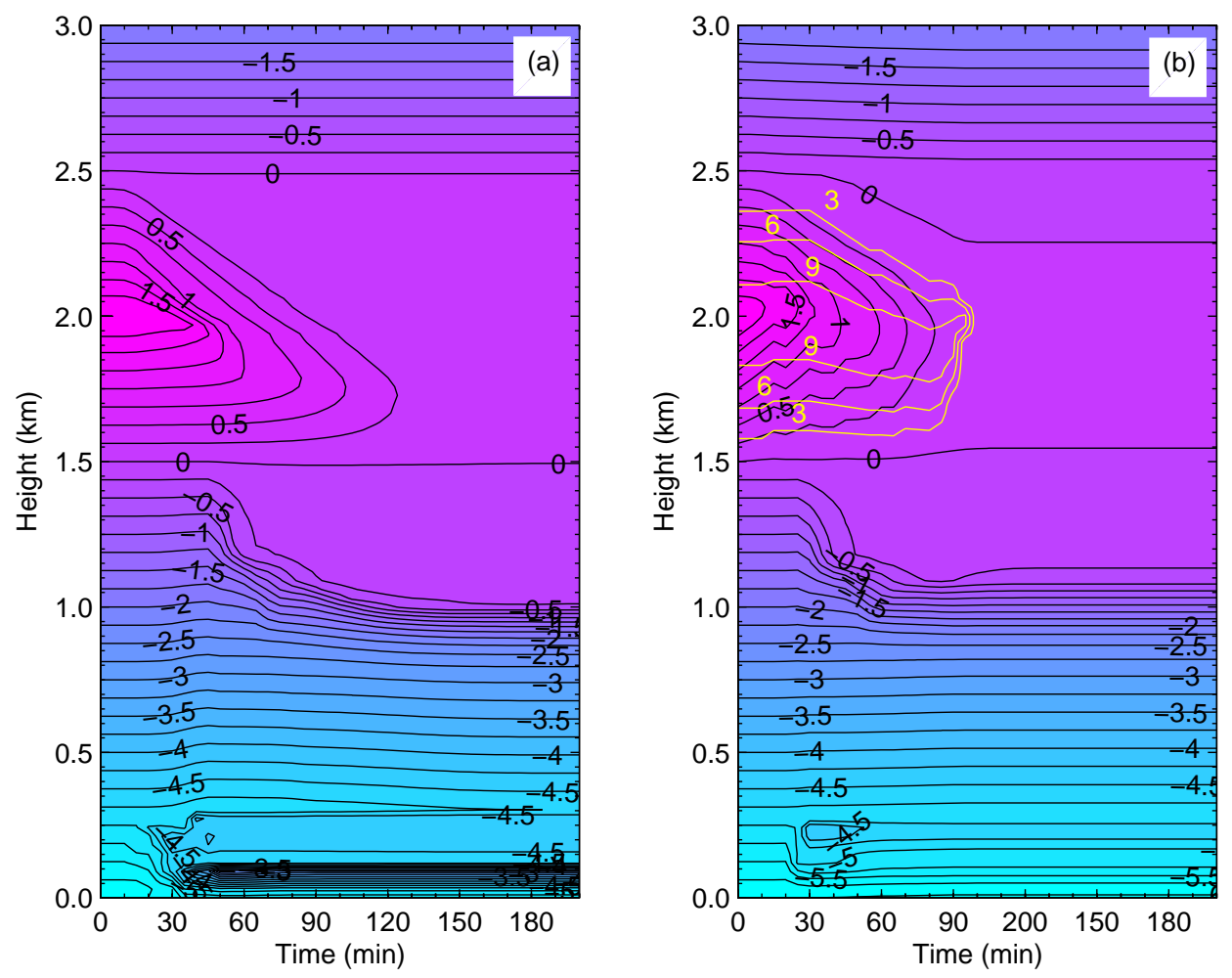

Fig. 4. The time evolution of the temperature profile initialized by the typical sounding described in Sect. 4. (a) is the temperature structure in still air and (b) is the temperature structure with a background ascending air $\left(w_{\max }=10 \mathrm{~cm} / \mathrm{s} \mathrm{in} \mathrm{Eq.} \mathrm{11).} \mathrm{The} \mathrm{black} \mathrm{lines} \mathrm{are} \mathrm{isotherms}\left({ }^{\circ} \mathrm{C}\right)\right.$ and the yellow lines in (b) indicate the vertical velocity structure $(\mathrm{cm} / \mathrm{s})$.

\section{Results}

\subsection{Vertical temperature profile evolution}

The time evolution of the vertical temperature profile has been investigated. Figure 4a illustrates results in still air and Fig. $4 b$ illustrates results with the effect of vertical air velocity. The variation of the temperature profile with time is caused by latent heat released or absorbed due to phase changes of the falling particles as well as by adiabatic cooling of the ascending air. Those features are explained in Sect. 2. Only the temperature is plotted on the diagram because, during the evolution, the atmosphere is at saturation.

As described in Sect. 3, the time evolution of the temperature begins with the typical temperature profile. It varies with time until the melting layer has completely evolved into an isothermal layer of $0^{\circ} \mathrm{C}$ mainly due to melting of snowflakes. At this point, snow falling from above no longer undergoes melting, and snow is the only precipitation type reaching the surface.

In Fig. 4, many temperature changes are observed in the melting and near the surface. However, the changes in the temperature profile above the melting layer $(>2.5 \mathrm{~km})$ with time are negligible because the atmosphere is saturated with respect to ice and only snow is falling in that part of the column. We will only discuss the change in temperature in the melting and subfreezing layers.

Many temperature variations occur in the melting layer. The temporal evolution of the temperature in the melting layer (between 1.5 and $2.5 \mathrm{~km}$ ) is very different between the case in still air and the one with the inclusion of background vertical air velocity. In still air (Fig. 4a), the isotherms are altered more in the upper region of the melting layer, indicating the formation of the isothermal layer of $0^{\circ} \mathrm{C}$ whereas very little change are observed in the lower part of the inversion. The decrease in temperature of the melting layer is therefore due to the melting of snowflakes that are falling through it. This implies that snowflakes melt when entering the warm region and that is why only the upper part of the melting layer is experiencing cooling. When vertical air velocity is considered (Fig. 4b), the isotherms in the melting layer evolve differently. The decrease in temperature is distributed over the entire layer due to the adiabatic cooling from ascending air prescribed in the cloud model. The effect is also coupled with the melting-by-cooling of snowflakes in the upper region of the melting layer as in Fig. 4a.

The time and rate at which the melting evolves into an isothermal layer of $0^{\circ} \mathrm{C}$ is very important. Figure 5 shows 
the evolution with time of the melting layer's depth in still air and with the background vertical air velocity. In still air, the depth of the melting layer decreases constantly until it has completely evolved into an isothermal layer of $0^{\circ} \mathrm{C}$ after $200 \mathrm{~min}$. However, because of the effect of adiabatic cooling, the depth of the melting layer in the case with a background vertical air velocity decreases faster until it quickly evolves into an isothermal layer of $0^{\circ} \mathrm{C}$ at $100 \mathrm{~min}$. Furthermore, in still air, the temperature decreases very rapidly in the melting layer until it gets very close to $0^{\circ} \mathrm{C}$ (Fig. 4a). For example, the maximum temperature of the melting layer reaches $0.25^{\circ} \mathrm{C}$ at $120 \mathrm{~min}$ and the melting layer is completely converted into an isothermal layer of $0^{\circ} \mathrm{C} 80 \mathrm{~min}$ later. Similar to the depth of the melting layer, the change in the temperature profile is very rapid with background vertical air velocity.

Finally, the temperature profile associated with the subfreezing layer below the melting layer also varies with time. In both cases (Figs. $4 \mathrm{a}$ and $\mathrm{b}$ ), the temperature begin to increase in the subfreezing layer just below the melting layer (between 1 and $1.5 \mathrm{~km}$ ). This warming is due to the formation of ice pellets in this region and this consequently warms the atmosphere. However, near the surface (between 0 and $0.3 \mathrm{~km}$ ), a large vertical temperature gradient is observed starting at $30 \mathrm{~min}$ in still air and this effect decreases with increasing background vertical air velocity. This is due to collisions of freezing raindrops with ice particles that result in the freezing of those drops and this in turn leads to the release of latent heat in the atmosphere.

\subsection{Precipitation types and cloud droplets in a vertical air} column

The temperature structure is closely related to the various types of precipitation formed in the atmosphere. The evolution of precipitation types formed when a background ascending air velocity is assumed is shown Fig. 6. This figure illustrates the mass content profile at three critical times based on the temperature profiles shown in Fig. 4. Those times have been chosen to show profiles near the beginning of the event $(30 \mathrm{~min})$, the middle of the event $(60 \mathrm{~min})$, and the time when the temperature inversion is almost completely eliminated (90 min).

At 30 min, many types of hydrometeors are formed in the melting layer (Fig. 6a) such as slush, wet snow, rain and cloud droplets. The cloud droplets are generated by condensation due to the cooling-by-melting process and the ascending velocity in the melting layer. In the scheme, cloud droplets are parameterized as a function of supersaturation and vertical air velocity. Freezing rain, slush, ice pellets and graupel are formed upon passage of particles through the refreezing layer below it. Graupel, for example, is produced by collisions of freezing rain and ice particles near the surface (Milbrandt and Yau, 2005b). Also, slush produced near the top of the refreezing layer (between 1 and $1.5 \mathrm{~km}$ ) freezes

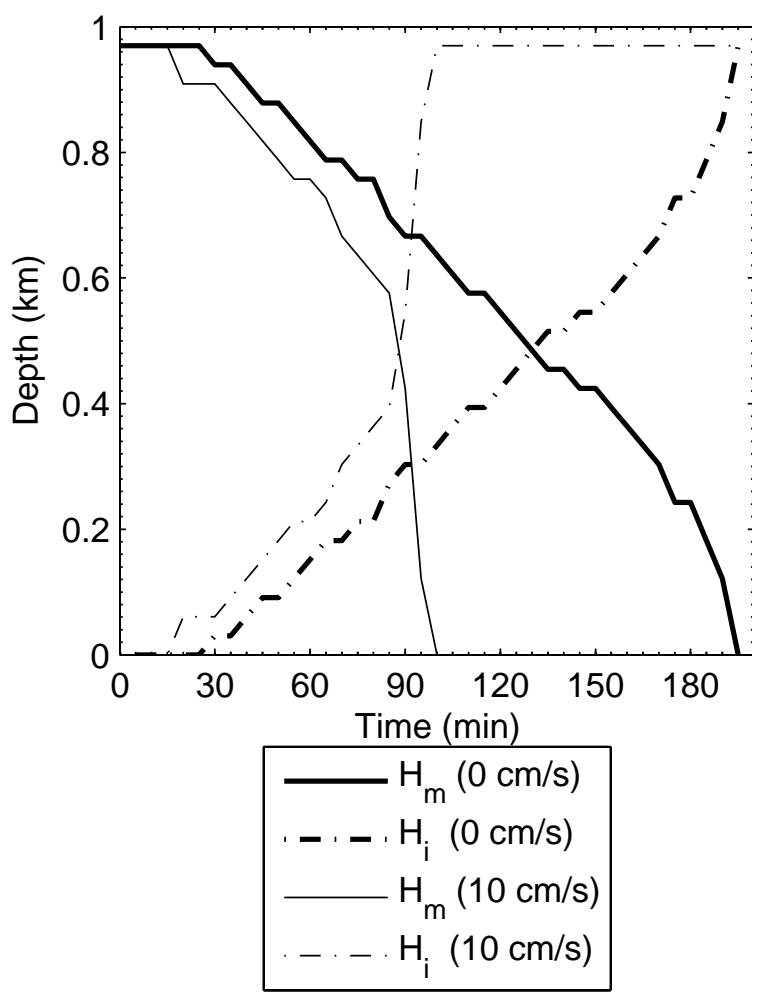

Fig. 5. The variation of the depth of the melting layer (solid lines) and the isothermal layer (dotted lines) in still air (bold) and with the effect of background vertical air velocity of $10 \mathrm{~cm} / \mathrm{s} . H_{m}$ refers to the depth of the melting layer and $H_{i}$ refers to the depth of the isothermal layer at $0^{\circ} \mathrm{C}$.

into ice pellets and this contributes to the warming of the atmosphere in this region (Fig. 4b).

At 60 min (Fig. 6b), snow is present at the top of the warm layer $(\mathrm{z}>2.3 \mathrm{~km})$ because an isothermal layer of $0^{\circ} \mathrm{C}$ has been formed. At this time, the melting layer is also slightly colder and snow is evolving into wet snow, slush and rain. These precipitation types will change into refrozen wet snow and ice pellets, respectively, in the subfreezing layer. Finally, many cloud droplets are still present but in a lower concentration than at $30 \mathrm{~min}$ since these are being swept out by larger precipitation particles.

When the melting layer is almost eliminated (Fig. 6c), the main precipitation type in the melting layer is wet snow with a small amount of slush, rain. There are also some cloud droplets. Thus, a combination of ice pellets and refrozen wet snow is present in the subfreezing layer. However, some cloud droplets are still present in the melting layer because of their continual regeneration by adiabatic cooling and cooling-by-melting. As well, the snow mass content demonstrates that the isothermal layer is deeper than at $60 \mathrm{~min}$.

In the third panel of Fig. 6, the mass content of snow decreases with height. This arises because of snowflake 

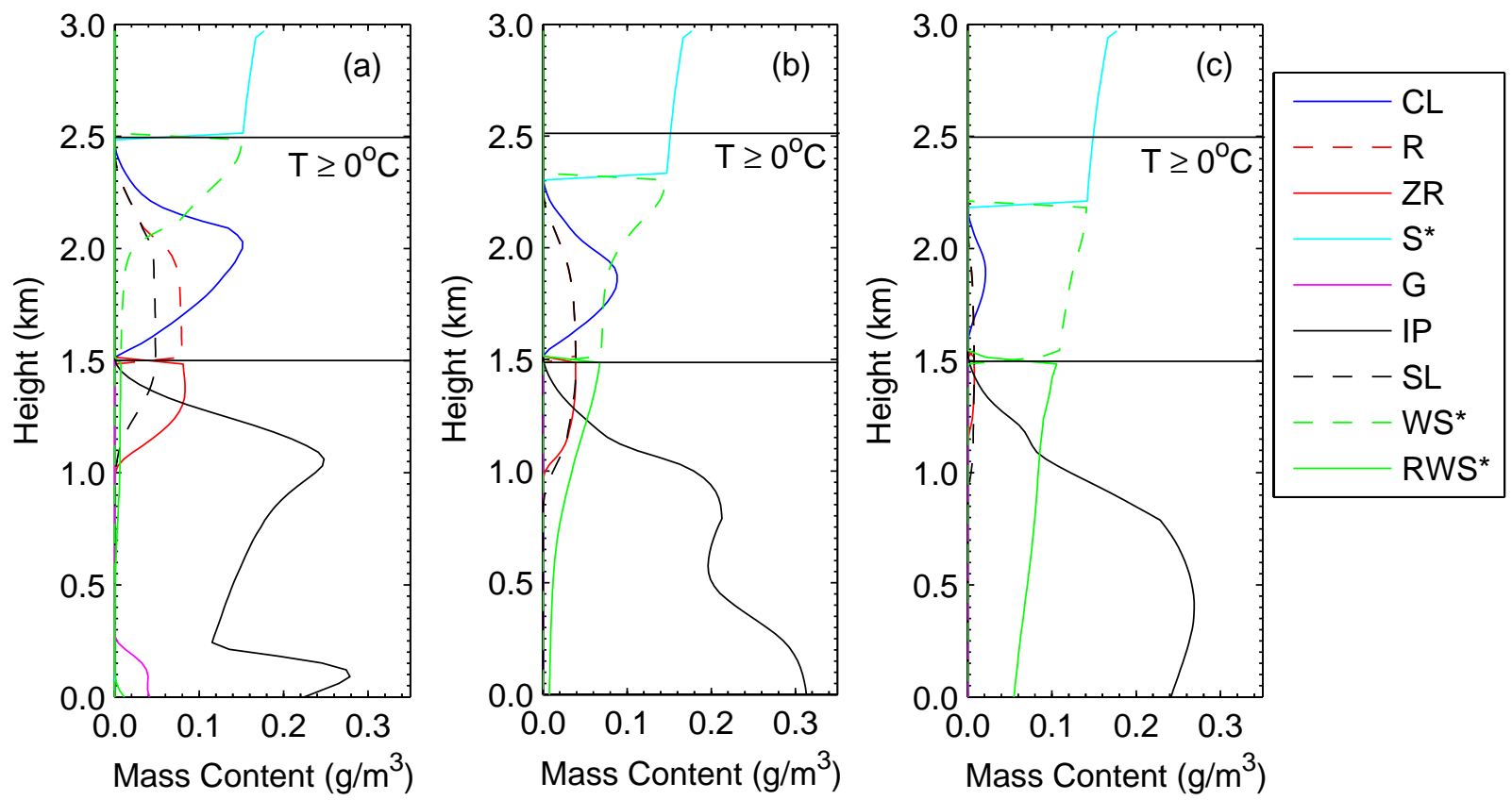

Fig. 6. The evolution of precipitation types and cloud droplets in gently ascending warm air is shown. The initial melting layer is between the two bold lines $\left(\mathrm{T} \geq 0^{\circ} \mathrm{C}\right.$ ). It shows the vertical evolution of the various precipitation types formed at (a) $30 \mathrm{~min}$, (b) $60 \mathrm{~min}$ and (c) $90 \mathrm{~min}$ in a $10 \mathrm{~cm} / \mathrm{s}$ ascending air. The symbols for the precipitation types are cloud droplets (CL), rain (R), freezing rain (ZR), snow (S), graupel $(\mathrm{G})$, ice pellets (IP), slush (SL), wet snow (WS) and refrozen wet snow (RWS). In the legend, * means that the mass content plotted is 10x smaller than the actual value.

aggregation and sedimentation. At temperatures $>-5^{\circ} \mathrm{C}$, snowflakes are more likely to stick together; this triggers a decrease in the total concentration and an increase in the snowflake's diameter. Also, temperatures near $0^{\circ} \mathrm{C}$ are favorable for aggregation and in our case deep isothermal layers of $0^{\circ} \mathrm{C}$ are generated by melting and act to increase the snowflake's diameter. In a double-moment microphysics scheme, larger snowflakes fall faster, thus the decrease in mass content is associated with the fewer but larger snowflakes that did not melt when falling through the melting layer.

Figure 7 shows the difference between the mass content of the various precipitation types formed in still air and in vertically ascending air. Positive values mean that more of this type of precipitation is present when background ascending air is considered and negative values mean that more of this precipitation type is present in still air.

At 30 min (Fig. 7a), more slush, cloud droplets and ice pellets are observed with background vertical air velocity whereas more freezing rain and rain are observed in still air through the entire column. A larger amount of graupel and ice pellets is present at the surface in still air than with the effect of vertical air velocity. Those particles are formed by freezing through collisions and this process warms the atmosphere near the surface as described in Fig. 4a.
At later times (Fig. 7b), more wet snow is present at the top of the warm layer in still air whereas snow is present with the effects of background vertical air velocity. This result shows that the isothermal layer of $0^{\circ} \mathrm{C}$ is deeper with the effects of ascending air than in still air. Also, snow, cloud droplets, wet snow and refrozen wet snow are more abundant with background vertical air velocity, whereas more ice pellets are formed in still air. The presence of more refrozen wet snow in the subfreezing layer with the effect of background vertical air velocity accounted for means the melting layer is colder than in still air, reducing the amount of melting. However, the main precipitation type in the refreezing layer in both cases is ice pellets.

Near the end of the evolution of the melting layer into a $0^{\circ} \mathrm{C}$ isothermal layer (Fig. $7 \mathrm{c}$ ), the temperature is very close to $0^{\circ} \mathrm{C}$, wet snow is the dominant precipitation type with background vertical air velocity whereas slush and rain are dominant in still air. The amount of ice pellets varies between positive and negative values in the subfreezing layer near the surface. The negative value of ice pellets in the subfreezing layer means that the melting layer is not completely eliminated at that time in still air. However, the mass content of ice pellets is increasing with height in the subfreezing layer. This means that most of the refrozen wet snow and ice pellets have almost reached the surface when the effect of vertical air velocity is considered whereas the maximum 

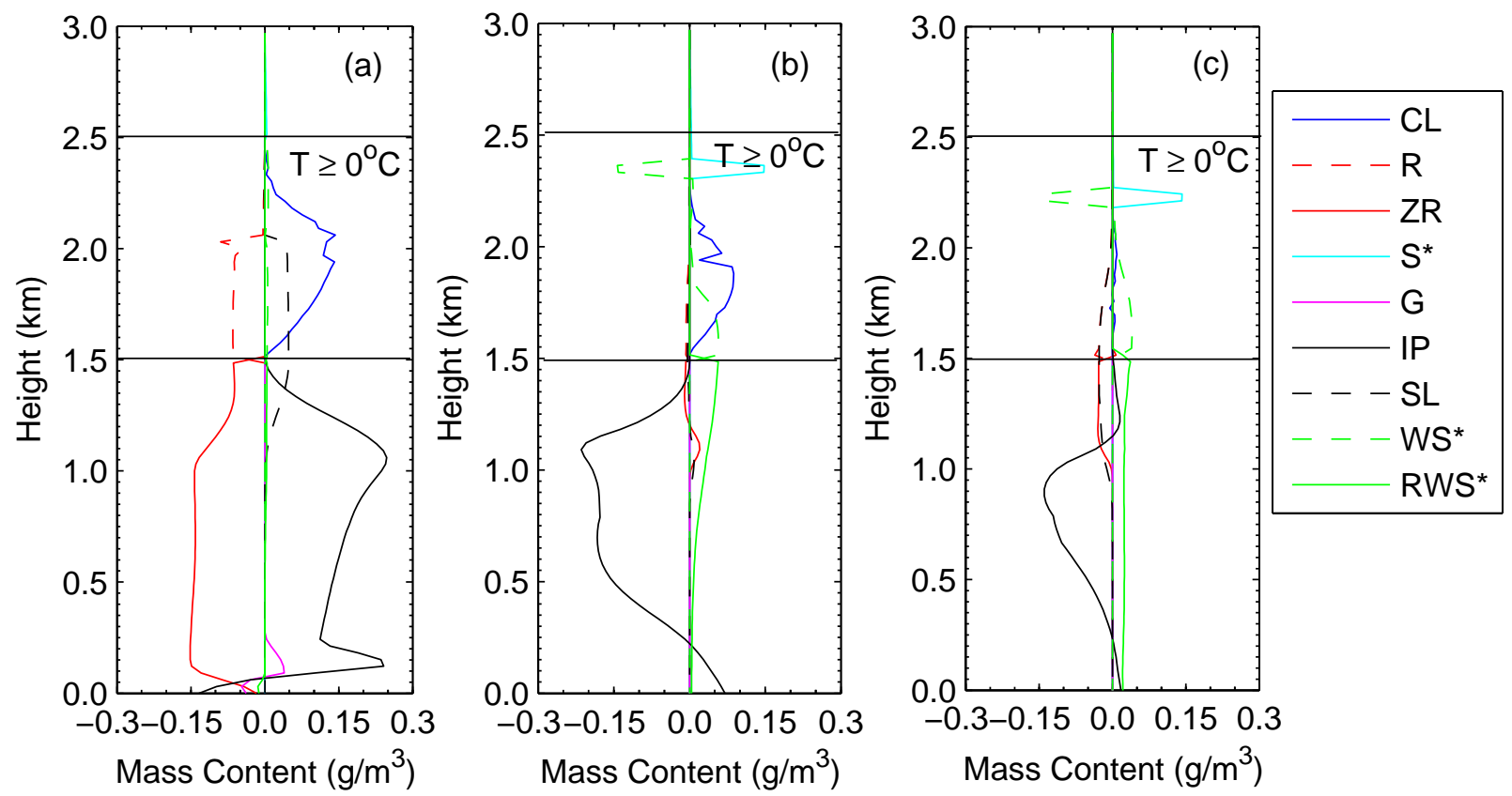

Fig. 7. The evolution of precipitation types and cloud droplets in gently ascending warm air and differences from a still air environment. The initial melting layer is between the two bold lines $\left(\mathrm{T} \geq 0^{\circ} \mathrm{C}\right)$. It shows the difference between the mass content of each precipitation types and cloud droplets between the situation with a background vertical ascent with $w_{\max }=10 \mathrm{~cm} / \mathrm{s}$ and the situation with still air for the same time interval. The symbols for the precipitation types are cloud droplets (CL), rain (R), freezing rain (ZR), snow (S), graupel (G), ice pellets (IP), slush (SL), wet snow (WS) and refrozen wet snow (RWS). In the legend, $*$ means that the mass content plotted is $10 \mathrm{x}$ smaller than the actual value.

value of those precipitation types is just below the melting layer in still air. Thus, the case of only snow reaching the surface occurs more rapidly when ascending air is considered in the melting layer.

\subsection{Precipitation types at the surface}

A detailed analysis of the temperature profile and the precipitation types formed in the air column has been carried out. This section focuses on the precipitation types reaching the surface. We will also investigate the impact of the strength of the vertical velocity on the surface accumulated precipitation.

Figure 8 shows the evolution of the various precipitation types reaching the surface when the model is initialized with the typical temperature and vertical air velocity profile defined in Fig. 3. A mixture of freezing rain, a trace of graupel and some refrozen wet snow reach the surface over the first $30 \mathrm{~min}$. Those precipitation types are followed by a longer period of ice pellets only, eventually combined with refrozen wet snow. Snow starts to reach the surface approximately 25 min after the melting layer has completely cooled to $0^{\circ} \mathrm{C}$.

The order in which each precipitation type reaches the surface is consistent with observations (Fig. 1). For example, starting near the surface front $(0 \mathrm{~km})$ and moving in the di- rection ahead of the front, the surface precipitation is changing from freezing rain and ice pellets into refrozen wet snow and snow. A deeper melting layer and shallow subfreezing layer is associated with freezing where the opposite is true for ice pellets (Zerr, 1997). When the melting layer is no longer present aloft, snow is reaching the surface.

On Fig. 8, we have also plotted the snow evolution with a weaker vertical air velocity $w_{\max }=5 \mathrm{~cm} / \mathrm{s}$ and in still air. The time at which the refrozen wet snow is changed into snow is much later in still air than with a vertical air velocity of $5 \mathrm{~cm} / \mathrm{s}(70 \mathrm{~min})$ and even longer with a vertical air velocity of $10 \mathrm{~cm} / \mathrm{s}(100 \mathrm{~min})$. That is the reason why time plotted is longer than in previous figures. Also, this implies that the time needed for the melting layer to evolve into an isothermal layer of $0^{\circ} \mathrm{C}$ increases with decreasing strength of the ascent rate of the warm air.

To illustrate the amount of each precipitation type reaching the ground, we show the accumulated precipitation at the surface over $240 \mathrm{~min}$ for various values of $w_{\max }$ (Fig. 9). It is demonstrated by a percentage of the total amount of precipitation in liquid equivalent reaching the surface for each different case. We have chosen 240 min because it takes a little less than this amount of time to have snow reaching the surface in still air. 


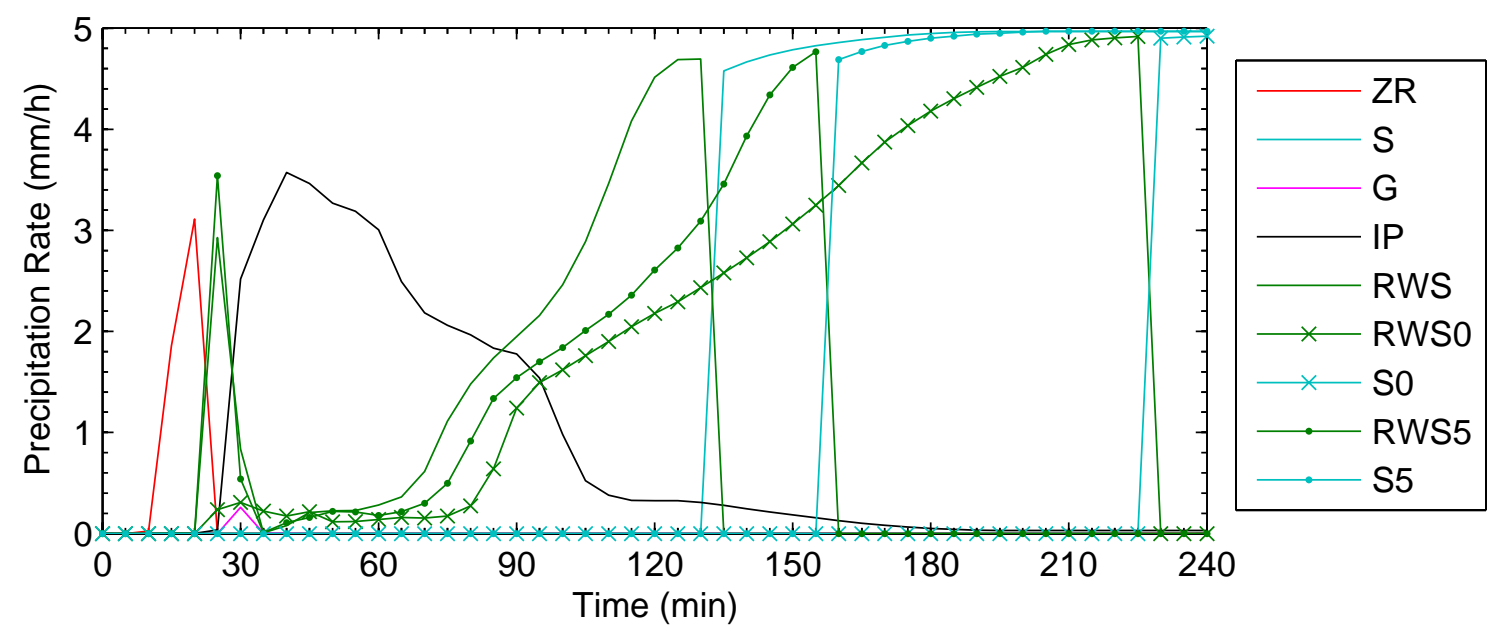

Fig. 8. The evolution of all the precipitation types are for $w_{\max }=10 \mathrm{~cm} / \mathrm{s}$ and the evolution of snow for $w_{\max }=0 \mathrm{~cm} / \mathrm{s}$ and $w_{\max }=5 \mathrm{~cm} / \mathrm{s}$. The symbols for the precipitation types are freezing rain (ZR), snow (S), graupel (G), ice pellets (IP), refrozen wet snow (RWS), refrozen wet snow (RWS5) and snow (S5) when $w_{\max }=5 \mathrm{~cm} / \mathrm{s} \mathrm{(Eq.} \mathrm{11),} \mathrm{refrozen} \mathrm{wet} \mathrm{snow} \mathrm{(RWS0)} \mathrm{and} \mathrm{snow} \mathrm{(S)} \mathrm{when} w_{\max }=0 \mathrm{~cm} / \mathrm{s}($ Eq. 11).

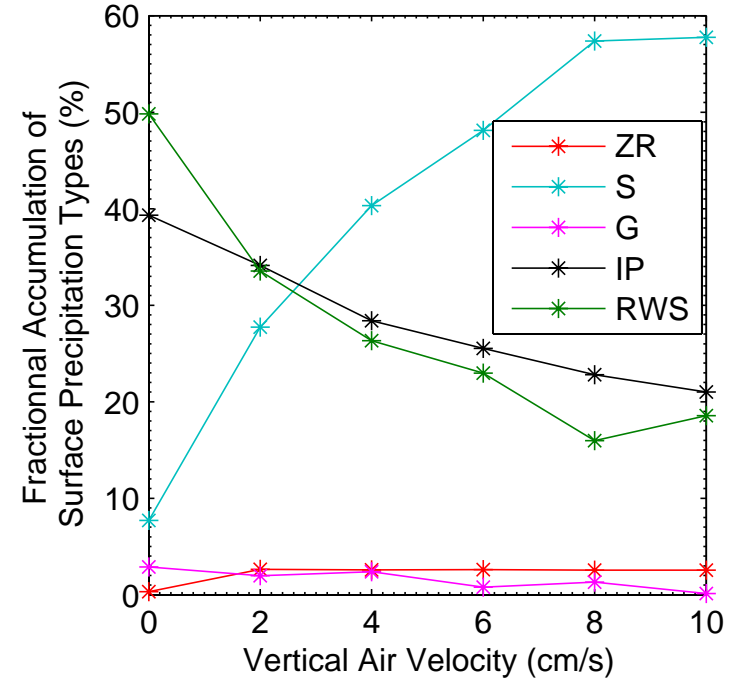

Fig. 9. The fractional accumulation (\%) of precipitation types at the surface through $240 \mathrm{~min}$ for various values of vertical air velocities $\left(w_{\max }\right)$. The symbols for the precipitation types are freezing rain (ZR), snow (S), graupel (G), ice pellets (IP) and refrozen wet snow (RWS).

A larger ascending air velocity is associated with more snow at the surface because the temperature inversion is eliminated faster. For example, the amount of snow reaching the surface is nearly $50 \%$ larger with a background vertical velocity of $10 \mathrm{~cm} / \mathrm{s}$ than in still air. In addition, the amount of other precipitation types such as ice pellets, refrozen wet snow and graupel at the surface decreases with increasing background vertical air velocity. For instance, the amount of ice pellets decreases by $18 \%$ when the vertical air velocity changes from $0 \mathrm{~cm} / \mathrm{s}$ to $10 \mathrm{~cm} / \mathrm{s}$ and refrozen wet snow by $31 \%$ in the same conditions.

Furthermore, Fig. 9 shows that essentially no freezing rain is observed in the case of still air whereas it increases slightly with background vertical air velocity. Freezing rain occurs at the beginning of the evolution with vertical air velocity due to the increase of cloud droplets being formed in the melting layer. This larger amount of cloud droplets leads to an increase of their collection by falling snowflakes in the melting layer. Because the cloud droplets have a temperature $>0^{\circ} \mathrm{C}$, this process increases the melting rate of snowflakes. Hence, more snowflakes melt completely and these form more freezing raindrops in the subfreezing region that have on average a larger mean diameter. Therefore, there is a greater likelihood of some of these many particles reaching the surface without being collected by ice particles. In still air, some freezing rain is produced aloft, as with effects of vertical air velocity, but these particles are eliminated near the surface by collisions with ice particles before reaching the surface.

Based on the results depicted in Figs. 8 and 9, many types of precipitation reach the surface in all cases but there are differences that depend on vertical air velocity. First, ice pellets only reach the surface first when there is still air (Thériault et al., 2006) and this situation is also the only one in which essentially no freezing rain is produced. Second, with vertical air velocity, the order at which the precipitation types occur as well as their starting times are similar. Third, the duration of most precipitation types at the surface decreases with increasing background vertical air velocity because the melting layer cools faster. Fourth, the "shape" of the precipitation rate of various types varies with vertical air velocity. For example, the refrozen wet snow precipitation rate at the 
surface increases faster in larger vertical air velocity that in still air.

\section{Conclusions}

A systematic study of the influence of background ascending air velocity associated with a winter storm transition region on the formation of winter precipitation types has been conducted. This transition region is often associated with a warm front. We simulate this synoptic feature in a simple manner by considering vertical air velocity in the warm air sector and neglecting horizontal advection. A one-dimensional kinematic cloud model coupled with a double-moment microphysics scheme is used (Thériault et al., 2006). The model is initialized by a typical temperature profile consisting of a melting layer aloft and a subfreezing layer in a saturated environment.

We showed that the evolution of the melting layer into one at $0^{\circ} \mathrm{C}$ is much faster with a background vertical air velocity than in still air. For example, it takes twice as long to produce such a temperature profile in still air than with a vertical air velocity of $10 \mathrm{~cm} / \mathrm{s}$ in the melting layer. This evolution is associated with a systematic evolution in the type of precipitation reaching the surface. Precipitation always evolves from those associated with at least partial melting into snow.

The background ascent also leads to major changes in the evolution of the temperature structure compared to the case of still air. In the melting layer, cooling is distributed over the whole layer with vertical ascending air as opposed to just its top initially in still air. The cooling effects also contributes to the production of supersaturated conditions in the melting layer. This phenomenon leads to the formation of a large amount of cloud droplets and this acts to warm the atmosphere. It also increases the collection of these cloud droplets by snowflakes and allows the formation of freezing rain at the surface at the beginning of the overall evolution by increasing their melting rate.

In the subfreezing layer near the surface, the change in temperature is more significant in still air than with ascending air. For example, near-surface (between 0 and $0.3 \mathrm{~km}$ ) temperatures $50 \mathrm{~min}$ into the simulations were up to $2.8^{\circ} \mathrm{C}$ warmer with still air in the melting layer than with ascent in this layer. This warming effect in still air is partly due to the latent heat release through freezing initiated by collisions of freezing rain and ice particles. With ascent, freezing rain is produced and this does not give rise to significant warming just above the surface through this process.

A background vertical air velocity always decreases some types of precipitation and increases others. In particular, the amount of ice pellets, refrozen wet snow and graupel decreases at the surface with increasing background air velocity whereas the amount of snow increases. Also, the refrozen wet snow precipitation rate increases more rapidly with increasing vertical air velocity. Finally, the detailed precipi- tation type evolution was similar in all cases although there were some distinct differences. For example, essentially no freezing rain is produced in still air.

We recognize that additional studies of this important topic are needed. A few examples of these issues are listed below. A first one is the effect of sub-saturation of the initial environment on reducing the melting rate. For example, Hanesiak and Stewart (1995) demonstrated that ice pellets can be enhanced by sub-saturation within the melting layer. A second issue is the horizontal temperature advection varying the temperature distribution in the atmosphere and directly affects the ensuing precipitation types. A third issue is the near surface warming of the air due to latent heat release from freezing of semi-melted particles and freezing rain upon impact with a sub-freezing surface. This warming can raise the surface temperature to $0^{\circ} \mathrm{C}$ and therefore eliminate the freezing process. A fourth issue is the incorporation of another type of precipitation into the model. In the present scheme, partially melted particles are considered but partially refrozen particles are not. Gibson and Stewart (2006) observed that ice pellets freeze from the outside inwards inside until they are completely frozen. These particles with an ice shell (we called them liquid core pellets) must play an important role in the sub-freezing region.

We demonstrated that very weak vertical air velocity $(\leq 10 \mathrm{~cm} / \mathrm{s})$ significantly influences the types and amount of precipitation formed in the atmosphere. The correct prediction of winter precipitation types therefore requires not only a detailed simulation of the evolution of precipitation particles but it also requires the careful simulation of the background air velocity. Therefore this study also leads to future research on the generation of these weak vertical velocities by frontal lifting as well as by diabatic heating and cooling driven by precipitation phase changes in a full three-dimensional context.

Acknowledgements. We would like to thank J. A. Milbrandt and M. K. Yau for their advice and for giving us the tool to conduct this research. We would like to thank the Natural Sciences and Engineering Research Council of Canada (NSERC), Environment Canada and the Institute for Catastrophic Loss Reduction (ICLR) for the financial support required to accomplish this work. One of us (JMT) would like to also thank NSERC for a post-graduate scholarship. The authors would like to thank three reviewers for their constructive comments on an earlier version of this article.

Edited by: A. Loukas

Reviewed by: K. Lagouvardos and two other referees

\section{References}

Findeisen, C.: The formation of the $0^{\circ} \mathrm{C}$ isothermal layer and fractocumulus under nimbostratus, Meteorol. Z., 54, 49-54, 1940.

Gibson, S. R. and Stewart, R. E.: Observations of ice pellets during a winter storm, Atmos. Res., doi:10.1016/j.atmosres.2006.11.004, 2006. 
Glickman, T. S.: Glossary of Meteorology, American Meteorology Society, 2nd edn., 2000.

Hanesiak, J. M. and Stewart, R. E.: The mesoscale and microscale of a severe ice pellets storm, Mon. Wea. Rev., 123, 3144-3162, 1995.

Henson, W., Stewart, R. E., and Kochtubajda, B.: On the precipitation and related features of the 1998 ice storm in Montréal area, Atmos. Res., 83, 36-54, 2007.

Milbrandt, J. A. and Yau, M. K.: A multi-moment bulk microphysics parameterization. Part I: Analysis of the role of the spectral shape parameter, J. Atmos. Sci., 62, 2051-3064, 2005a.

Milbrandt, J. A. and Yau, M. K.: A multi-moment bulk microphysics parameterization. Part II: A proposed three-moment closure and scheme description, J. Atmos. Sci., 62, 3065-3081, 2005 b.

Stewart, R. E.: Precipitation types in the transition region of winter storms, Bull. Am. Meteorol. Soc, 73, 287-296, 1992.
Stewart, R. E. and Macpherson, S. R.: Winter storm structure and melting-induced circulations, Atmos. Ocean, 27, 5-23, 1989.

Stewart, R. E., Yui, D. T., Chung, K. K., Hudak, D. R., Lezowski, E. P., Oleskiw, M., Sheppard, B. E., and Szeto, K. K.: Weather conditions associated with the passage of precipitation type transition regions over Eastern Newfoundland, Atmos. Ocean, 33, 25-53, 1995.

Szeto, K. K. and Stewart, R. E.: Effects of melting on frontogenesis, J. Atmos. Sci., 54, 689-702, 1997.

Thériault, J. M., Stewart, R. E., Milbrandt, J. A., and Yau, M. K.: On the simulation of winter precipitation types, J. Geophys. Res., 111, D18202, doi:10.1029/2005JD006665, 2006.

Wagner, J. A.: Mean temperature from $1000 \mathrm{mb}$ to $500 \mathrm{mb}$ as a predictor of precipitation type, Bull. Am. Meteorol. Soc., 38, 584590, 1957.

Zerr, R. J.: Freezing rain: An observational and theoretical study, J. Appl. Meteorol., 36, 1647-1661, 1997. 